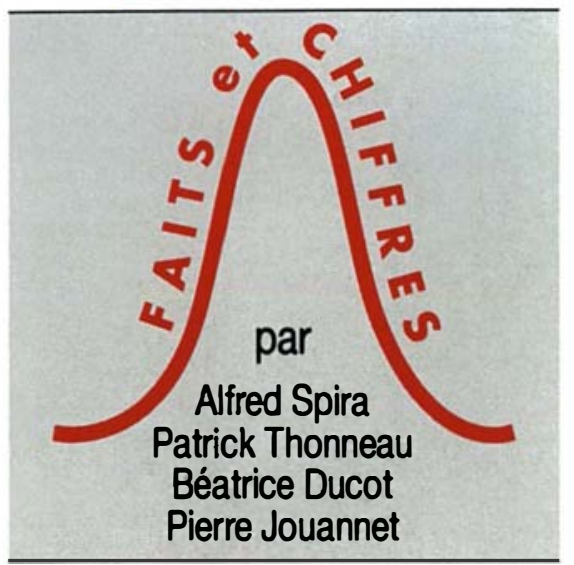

médecine/sciences $1995 ; 11: 621-2$

\section{RÉFÉRENCES}

1. Carlsen E, Giwercman A, Keiding N, Skakkebaek NE. Evidence for the decreasing quality of semen during the past 50 years. Br Med 1992 ; 305 : 609-13.

2. Auger J, Kunstmann JM, Czyglik F, Jouannet $\mathbf{P}$. Decline in semen quality of fertile men during the last 20 years. $N E n g l$ JMed 1995 ; 332 : 281-5.

\section{Alfred Spira \\ Patrick Thonneau \\ Béatrice Ducot \\ Inserm U. 292, hôpital de Bicêtre, 78, rue du Général Leclerc, 94275 Le Kremlin-Bicêtre Cedex, France.}

\section{Pierre Jouannet}

Laboratoire de biologie de la reproduction, hôpital Cochin-Port-Royal, 123, boulevard de Port-Royal, 75014 Paris, France.

\section{TIRÉS À PART}

A. Spira.

$m / s n^{\circ} 4$, vol. 11, avril 95

\title{
La qualité du sperme a-t-elle baissé au cours des cinquante dernières années?
}

D e toutes les excrétions humaines, c'est peut-être le sperme qui a suscité la plus grande attention, cela au moins pour deux raisons. D'une part, les spermatozoïdes sont des cellules gonadiques en production permanente, qui sont donc censées constituer un excellent reflet des fonctions de reproduction de l'individu et de leurs éventuelles modifications sous l'influence de facteurs intrinsèques ou extrinsèques. D'autre part, le sperme est (relativement) facile à recueillir puisque la masturbation est une pratique très largement répandue et il est alors aisé de compter les spermatozoïdes, cellules isolées et mobiles, ainsi que de mesurer leurs qualités et leur morphologie. Depuis que la méthodologie d'examen et d'analyse du sperme a été décrite par Hotchkiss en 1938 puis par Mac Leod en 1945, de très nombreuses publications ont rapporté les valeurs du nombre de spermatozoïdes par $\mathrm{ml}$ de liquide séminal dans des échantillons d'hommes dits "normaux " constitués dans différents lieux à diverses époques. De façon épisodique, de telles études ont suggéré la possibilité de l'existence, en dehors de toute pathologie, d'une altération au cours du temps de la qualité du sperme, c'est-à-dire en réalité d'une diminution de la concentration en spermatozoïdes des échantillons étudiés (maris de femmes enceintes, candidats au don de sperme, volontaires féconds, etc.), ainsi que d'altérations de leur mobilité et de leur morphologie. De façon à tenter de savoir si une telle tendance à la baisse existe réellement ou si elle n'est que le reflet de problèmes méthodologiques particuliers (biais de sélection différentielle, évolution dans les techniques de mesures), une étude systématique des données de la littérature a été effectuée par Carlsen et al. [1]. A partir d'une recherche bibliographique exhaustive, ces auteurs ont sélectionné 61 publications. Leur analyse montre (figure 1) une diminution significative de la concentration en spermatozoïdes dont la moyenne passe de $113 \mathrm{mil}$ lions/ml en 1944 à 66 millions/ml en 1990, ainsi que du volume séminal, de $3,40 \mathrm{ml}$ à $2,75 \mathrm{ml}$ durant la même période. Ils tentent de démontrer que cette baisse séculaire ne peut pas être expliquée par des problèmes de méthodes, et que donc, mises en parallèle avec l'augmentation observée durant la même période de l'incidence d'anomalies gonadiques telles que les cancers du testicule, la cryptorchidie et l'hypospadias, ces constatations pourraient être la conséquence de modifications de l'environnement plutôt que de facteurs génétiques. Ils évoquent en particulier l'hypothèse selon laquelle l'augmentation des dérivés œstrogéniques ou ayant une action de type œstrogénique dans les nutriments et l'eau de boisson pourrait être à l'origine de ces modifications, par imprégnation durant la vie fotale.

De nombreux débats, méthodologiques en particulier, ont agité la communauté scientifique autour de ces résultats, leur signification et

‥




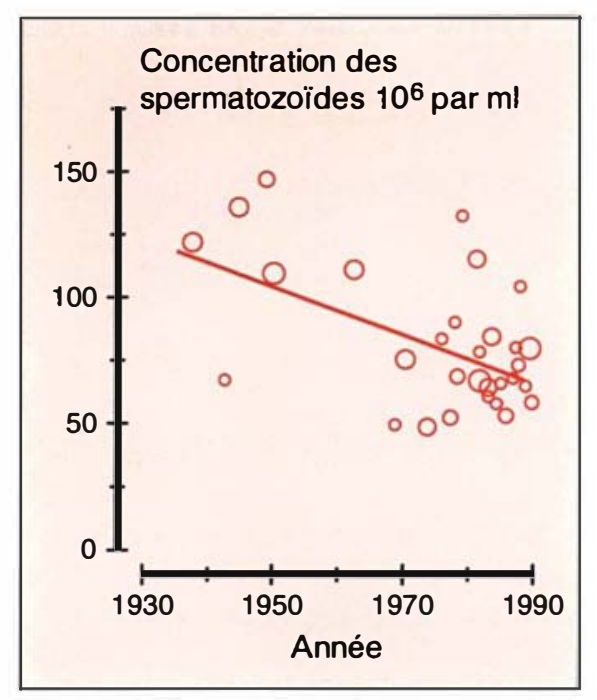

Figure 1. Évolution de la concentration du sperme en spermatozoïdes de 1938 à 1990 , dans le monde. Ce graphique rapporte les résultats de 61 publications provenant dans leur grande majorité des États-Unis et des pays occidentaux, mais avec aussi de grandes séries de Chine, de Lybie et du Brésil. La surface des cercles est proportionnelle au logarithme du nombre de sujets inclus dans l'étude [1].

leurs conséquences éventuelles posant véritablement un problème. La publication récente par l'équipe du CECOS de Cochin d'une étude importante confirmant cette baisse séculaire a permis de faire considérablement avancer ce problème [2] Réalisée dans des conditions méthodologiques rigoureuses, elle montre clairement (figure 2) que la concentration spermatique moyenne des donneurs de sperme accueillis dans le même centre a diminué de 2,1\% par an, de $89.10^{6}$ par ml en 1973 à $60.10^{6}$ par $\mathrm{ml}$ en $1992(\mathrm{p}<0,001)$. Pendant la même période, les proportions de spermatozoïdes mobiles et de formes normales ont diminué respectivement de $0,6 \%$ et $0,5 \%$

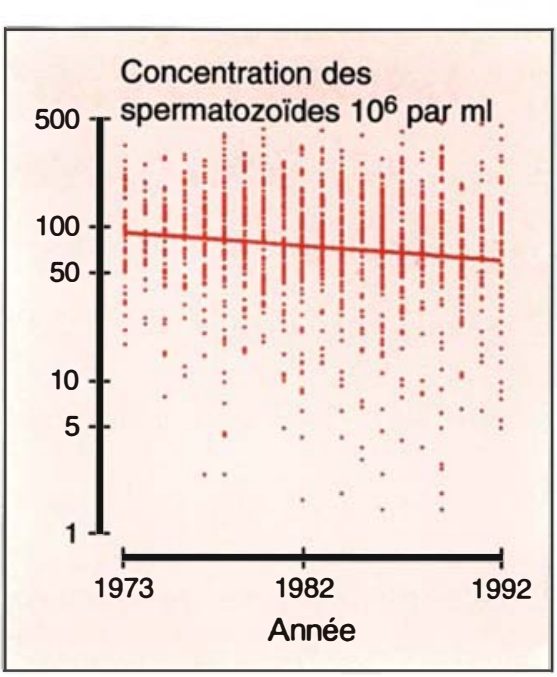

Figure 2. Évolution de la concentration du sperme en spermatozoïdes de 1973 à $1992 \mathrm{chez}$ les donneurs de sperme du CECOS de Bicêtre. L'analyse de la régression linéaire montre que la concentration moyenne du sperme a baissé de $2,1 \%$ par an, de $89.10^{6}$ par $\mathrm{ml}$ en 1973 à $60.10^{6}$ par $\mathrm{ml}$ en 1992. Pendant la même période, les proportions de spermatozoïdes mobiles et de formes normales ont diminué respectivement de 0,6\% et $0,5 \%$ par an.

te, qui est indépendante de l'âge, amène à envisager la possibilité que des modifications de l'environnement, de la nutrition ou des modes de vie puissent agir directement sur la production gamétique masculine. Sans que cela n'affecte directement la fécondité de façon notable, on ne peut pas exclure que l'ampleur du phénomène constitue un véritable signal d'alarme. Il paraît souhaitable maintenant d'effectuer des études permettant de mettre en évidence et d'évaluer les facteurs potentiellement incriminés. Ces derniers, et en particulier ceux concernant l'environnement professionnel, font actuellement l'objet d'études dans le cadre de l'action concertée européenne 
médecine/sciences $1995 ; 11: 623-4$

Les nouvelles

de ce numéro

ont été préparées par:

Valérie Allamand ${ }^{(1)}$

Jacques S. Beckmann ${ }^{(1,3)}$

Nathalie Bourg (1)

Lydie Brenguier ${ }^{(1)}$

Odile Broux ${ }^{(1)}$

Elisabeth Bursaux

Pierre Castelnau (2)

Nucharnard

Chiannilkulchai (1)

Daniel Cohen (3)

Erick Denamur ${ }^{(4)}$

Catherine Devaud (1)

Jean-Claude Dreyfus

Michel Fardeau (5)

François Fougerousse ${ }^{(1)}$

Hélène Gilgenkrantz ${ }^{(6)}$

Jean Gosselin ${ }^{(7)}$

Dominique Hillaire ${ }^{(1)}$

Charles E. Jackson (8)

Axel Kahn

Dominique Labie ${ }^{(6)}$

Brigitte Le Magueresse-

Battistoni (9)

Maria-Rita

Passos-Bueno (11)

Patricia Pasturaud (1)

Isabelle Richard ${ }^{(1)}$

Carinne Roudault ${ }^{(1)}$

Jay A. Tischfield (10)

Mireille Vasseur-Cognet ${ }^{(6)}$

Mayana Zatz ${ }^{(11)}$

\section{SOMMAIRE DES NOUVELLES BRÈVES}

L'effet antileucémique d'un inhibiteur de tyrosine kinase ciblé vers une protéine membranaire (p.626).

Une stimulation de l'activité de la protéine kinase CK2 constitue probablement I'un des mécanismes pathogéniques de la theilériose (p. 629).

Membrane érythrocytaire et croissance du Plasmodium falciparum (p. 629).

Surdité liée à $I^{\prime} X$ par mutation du gène POU3F4 (p.630).

La dystrophine: une protéine impliquée dans la rigidité de la membrane musculaire (p. 630).

L'ADN de dinosaure est accueilli avec septicisme (p.631).

Un système de réponse transcriptionnelle au glucose chez les mammifères (p.631).

L'axe hypothalamo-hypophyso-surrénalien est essentiel au cours du développement, mais non dans la vie adulte... au moins chez les rongeurs (p.632).

Le gène scid semble coder pour une protéine kinase dépendante de I'ADN (p.633).
Drépanocytose: I'adhérence des réticulocytes SS à I'endothélium vasculaire, responsable des vaso-occlusions, est spécifique (p. 633).

Le gène de I'hypoplasie congénitale des surrénales (p.634).

Les nouveaux partenaires de $\mathrm{Bcl}-2$ et la régulation de l'apoptose (p. 635).

Une greffe de moelle osseuse protège de l'athérosclérose des souris déficientes en apolipoprotéine $E$ (p. 635).

De l'égoïsme plasmidique à la cartographie des génomes (p. 636).

$P C-1$, un inhibiteur endogène de l'action de l'insuline (p.636).

La sclérose tubéreuse de Bourneville humaine et un cancer rénal héréditaire du rat: un même gène (p.636).

Expression du récepteur de type I du FGF basique au cours du développement testiculaire du rat (p. 642).

Activation transcriptionnelle par protéolyse dépendante du signal (p. 642).

\section{L'antigène CD21 est-il l'unique récepteur du virus Epstein-Barr?}

Le virus d'Epstein-Barr (EB), appartenant à la famille des Herpesviridae, est l'agent causal de la mononucléose infectieuse. Il est aussi associé à deux types de cancers, le lymphome de Burkitt et le carcinome du nasopharynx. Le virus ayant infecté les cellules épithéliales de l'oropharynx, c'est là que débute la réplication virale. Présents en grand nombre dans l'oropharynx, les lymphocytes B sont également infectés. L'adsorp- tion du virus sur la membrane de la cellule cible est une étape cruciale du processus infectieux. Les lymphocytes B (et les cellules épithéliales de l'oropharynx) lient le virus EB via une glycoprotéine de $145 \mathrm{kDa}$ appelée CR2 ou CD21. Le CD21 est un récepteur ayant une double affinité, l'une pour le virus EB, l'autre pour le fragment C3d du complément. L'adsorption du virus sur le CD21 fait intervenir la gp350/220, une gly-

(9) Inserm U. 407, bâtiment 3B, Centre Hospitalier Lyon Sud, 69495 Pierre-Bénite Cedex, France.

(6) Inserm U. 129, CHU Cochin, 24, rue du Faubourg-Saint-Jacques, 75014 Paris, France.

(7) Laboratoire d'immunologie virale, centre de recherche en rhumatologie et immunologie, centre de recherche du CHUL et département de physiologie, Université Laval, 2705 , boulevard Laurier, Québec, Canada, GIV 4G2, Canavard

(8) Henry Ford Hospital, Detroit, MI 48202, ÉtatsUnis.
(10) Departement of medical and molecular ge netics, Indiana University School of Medicine 975 West Walnut Street, Indianapolis, IN 46202 5251, États-Unis.

(11) Department de biologia, Instituto de bio cièncias, Universidad de Sào Paulo, Brazil.

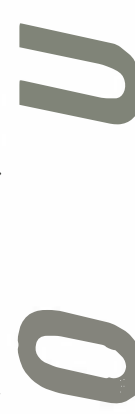


coprotéine fortement exprimée sur l'enveloppe externe du virus.

Bien que les lymphocytes B soient depuis longtemps reconnus comme la principale cible du virus EB, plusieurs études indiquent que ce virus peut aussi se lier à la surface membranaire d'autres types cellulaires. Par exemple, il a été démontré que les thymocytes immatures et les lymphocytes $\mathrm{T}$ pouvaient également être infectés par le virus EB [1]. En effet, la présence du génome viral et des antigènes EBNA-1-2* et LMP** a été détectée chez des malades ayant développé des lymphomes de type $\mathrm{T}$. Des études ultérieures ont suggéré qu'une ou plusieurs molécules différentes de l'antigène CD21 seraient reconnues par le virus EB. Ainsi, dans une étude portant sur des lymphocytes $\mathrm{T}$ humains fraîchement isolés, il a été démontré que le virus $\mathrm{EB}$ pouvait se lier à environ $50 \%$ des cellules $\mathrm{T}$ de phénotype $\mathrm{CD}^{+}$, bien que la présence du récepteur CD21 n'ait pu être détectée sur ces cellules par l'utilisation d'anticorps monoclonaux anti-CD21 [2]. Dans une étude récente, Hedrick et al. [3] proposent qu'une protéine de $70 \mathrm{kDa}$ agisse comme récepteur du virus $\mathrm{EB}$ à la surface membranaire des cellules HSB-2 (lignée cellulaire T) dépourvues de CD21, pour lesquelles, cependant, on a montré liaison virale et infection. Dans cette étude, les auteurs ont produit une protéine recombinante correspondant aux 470 premiers acides aminés de la portion N-terminale de la gp350/ 220, protéine de l'enveloppe du virus EB qui pouvait se lier à la membrane des cellules HSB-2 et Raji et inhiber la liaison du virus à ces cellules. Afin de déterminer le poids moléculaire de l'antigène membranaire reconnu par la protéine recombinante, des homogénats de cellules HSB-2 ont été soumis à une étape de purification par chromatographie d'affinité en utilisant la protéine recombinante comme matrice. L'analyse par électrophorèse en gel de polyacrylamide en présence du détergent SDS (sodium dodecyl sulfate)

\footnotetext{
* EBNA : Epstein-Barr nuclear antigen.

** LMP : latent membrane protein.

*** EA : early antigen
}

a révélé que la protéine isolée avait une masse moléculaire de $70 \mathrm{kDa}$, différente donc de celle du récepteur CD21 déjà connu (145kDa), suggérant ainsi qu'il existe plus d'un récepteur du virus $\mathrm{EB}$.

En outre, Cantaloube et al. [4] avaient proposé qu'une molécule autre que le récepteur CD21 joue un rôle important dans l'infection du lymphocyte B par le virus EB à l'étape de la fusion des membranes, permettant l'internalisation de la capside dans la cellule. Utilisant des fibroblastes de souris transfectés avec de l'ADN génomique humain, ces auteurs ont pu produire des clones exprimant l'antigène CD21. Bien que le récepteur CD21 exprimé à la surface des cellules transfectées se soit révélé fonctionnel, tant pour la liaison du virus EB que pour la liaison du fragment C3d, aucun gène viral (EBNA, EA*** et $\left.\mathrm{VCA}^{* * * *}\right)$ ne put être détecté par immunofluorescence, même après plusieurs jours de culture en présence de particules virales. Les auteurs ont donc conclu que la présence du CD21 était insuffisante à la pénétration du virus, suggérant qu'un ou des facteurs additionnels étaient nécessaires au processus infectieux. Tel est le cas pour le virus Herpes simplex et probablement pour le virus de l'immunodéficience acquise humaine, où une deuxième molécule serait impliquée dans l'internalisation du virus. Il en est de même pour les adénovirus qui utilisent un récepteur pour se lier à la cellule et, comme deuxième récepteur pour pénétrer la cellule cible, une molécule de la famille des intégrines [5].

L'ensemble de ces données, d'une part, démontre que les lymphocytes B (et les cellules épithéliales de l'oropharynx) ne sont pas les cibles exclusives du virus $E B$ et que le virus EB peut se lier à des récepteurs autres que CD21 et, d'autre part, suggère qu'en plus des récepteurs responsables de la liaison du virus à la cellule, d'autres molécules pourraient être impliquées dans le processus d'internalisation. Ces données récentes incitent à considérer que le virus EB pourrait avoir des cibles cellulaires et moléculaires non reconnues à ce jour.
1. Tsoukas CD, Lambris JD. Expression of EBV/C3d receptors on T cells : biological significance. Immunol Today 1993; 14: 56-9.

2. Sauvageau G, Stocco R, Kasparian S, Menezes J. Epstein-Barr virus receptor expression on human $\mathrm{CD8}^{+}$(cytotoxic/suppressor) $\mathrm{T}$ lymphocytes. J Gen Virol 1990; 71 : 379-86.

3. Hedrick JA, Lao Z, Lipps SG, Wang X, Todd SC, Lambris JD, Tsoukas CD. Characterization of a 70-kDa, EBV gp 350/220-binding protein on HSB-2 T cells. J Immunol 1994; $153: 4418-26$.

4. Cantaloube JF, Piechaczyk M, Calender A, Lenoir G, Minty A, Carrière D, Fisher E, Poncelet $P$. Stable expression and function of EBV/C3d receptor following genomic transfection into murine fibroblast L cells. Eur J Immunol $1990 ; 20$ : 409-16.

5. White J. Integrins as virus receptor. Curr Biol 1993; 3 : 596-9. 